\title{
Expanding Usability of Virtual Network Laboratory in IT Engineering Education
}

\author{
http://dx.doi.org/10.3991/ijoe.v9i1.2388 \\ Dalibor Dobrilovic, Vesna Jevtic, Borislav Odadzic \\ University of Novi Sad, Zrenjanin, Serbia
}

\begin{abstract}
This paper deals with importance of virtual network laboratories usage in IT engineering education. It presents the particular virtual network laboratory model developed for usage in Computer Networks course as well. This virtual network laboratory, called VNLab, is based on virtualization technology. It has been successfully tested in educational process of Computer Network course for IT undergraduate students. Its usability for network related courses is analyzed by comparison of recommended curricula's of world organizations such as IEEE, ACM and AIS. This paper is focused on expanding the usability of this virtual network laboratory to other non-network related courses. The primary expansion field is in domain of IT System Administration, IT Systems and Data Security and Operating Systems as well. The possible learning scenarios, learning tools and concepts for making this system applicable in these three additional fields are presented by the analyses of compatibility with recommended learning topics and outcomes by IEEE, ACM and AIS.
\end{abstract}

Index terms-component; Computer Network course; System Security course, System Administration course, engineering education; virtual network laboratories; virtualization technology

\section{INTRODUCTION}

There are numerous ways for adopting practical and functional knowledge about computer networks and networked systems. The first one is to use laboratories with real network equipment, which enable students to work in real network surrounding. The problems that can occur in this case are laboratories' cost and availability. Laboratories with real network equipment also have limited access, considering the simultaneous work for certain group of students.

The second way of teaching practice can help to overcome these problems. For example, software tools for network modeling and simulation can be used. They have simulated environment designed for analysis and behavior prediction of Information and Communication Technology Infrastructures, including applications, servers and network technologies. Simulation software tools available nowadays are:

- OPNET IT Guru software is discrete event network simulator in computer networks that support large number of simulations and scenarios for educational and commercial usage. OPNET IT Guru Academic edition is used at more than 500 universities for research and teaching purposes. Its commercial version is used in thousands of enterprises and government institutions all over the. [1]
- Ns-2 (Network Simulator) provides simulation interface for TCP/IP networks [2], routing and multicast protocols over wired and wireless networks. Ns-3 [1, $3,4]$ is free discrete event network simulator [5] that is used for networking research and education.

Apart from above mentioned tools there are also: Bosom NetSim, Tetcos NetSim, OMNeT++, GNS-3, Cisco Packet Tracer and others.

This paper concerns the third approach in teaching the computer networking concepts. It has emerged in the last several years and suggested virtual network laboratories based on virtualization technology. Its rapid development is closely related to the virtualization software breakthrough on the market in the last few years. [6]

The virtual network laboratory based on virtualization technology, called VNLab, is designed at Technical Faculty "Mihajlo Pupin" Zrenjanin (University of Novi Sad, Serbia) in order to meet the requirements in teaching networking concepts and to be used in Computer Network course. The usability of VNLab for that purpose is precisely determined in the initially research [7].

This paper addresses the expansion of VNLab usability in other courses in Computer Science fields, not strictly related to computer networks, by using the similar methodology. Methodology for determining the VNLab extended usability is based on comparison with courses related to recommended curricula's of international organizations such as IEEE, ACM and AIS. These three organizations have recommended five determined areas in the field of Computer Science such as: Computer Engineering, Computer Science, Information Technology, Software Engineering and Information Systems.

The main motivation for this research is to find effective and low cost solutions for teaching future Computer Science experts at higher educational institutions, applicable in more courses beyond the computer networks.

This paper presents the model of virtual network laboratory (named VNLab) with remote access based on virtualization technology, as well as description of the laboratory exercises, specially developed for this environment. At the end of the paper, the assessment of its expanded usage and support for other courses, beyond the network related ones is presented as well.

\section{VIRTUAL NETWORK LABORATORIES}

The virtual network laboratories and its features are briefly explained in this section. Virtualization software proved itself as efficient enough for virtual laboratories creation $[8,9,10,11]$. These laboratories have multiple applications. The basic one is to create experimentation 
environment for new technologies, as well as developing and testing of network software. The next important application of virtual laboratories is in education of IT experts, i.e. for engineering education.

Virtual laboratories can be used in the fields of: operating systems [12], system administration and networks security $[13,14,15,16]$, server and client software development, data bases [17], distributed network platforms and other [18]. The most popular use of virtual laboratories is in the field of computer network education. There are many different platforms developed for those purposes, as well as the ones primarily designed for experimenting and after for networking concepts studying.

Using both virtualization technology and appropriate software, virtual network environments, which completely corresponds to the real system, can be created. Virtualization enables creation of several virtual computers with different operating systems on one physical machine. Virtual computers can be servers, working stations and network units, such as router. Router's functionality can be totally emulated by virtual machine (VM) and specified software. Every virtual computer, i.e. virtual machine uses hardware of its physical computer. As every hardware component of the host computer, network interface card (NIC) is emulated too. Based on one physical card virtual machine can emulate many virtual network cards. There can be virtual connection between virtual machines and by that virtual network cards are connected in virtual network or its segment.

There is a lot of successfully created virtual network laboratories based on virtualization technology used for testing environments and in educational process: VNetLab [19], VLabNet [20, 21], GINI [22], VELNET (Virtual Environment for Learning Networking) [23, 24], Marionnet [25], vBET system [26], Dynagen [27].

\section{ARCHITECTURE OF VNLAB}

VNLab is virtual network laboratory with remote access. It is also an online educational system that distributes learning material, as well as learning environment, using the network infrastructure. The laboratory is based on virtualization technology.

VNLab was developed during 2007 at Technical Faculty "Mihajlo Pupin", University of Novi Sad, Serbia. It is used as teaching environment for Computer Network course for bachelor students at Information technology study program.

VNLab represents hardware-software model of virtual network laboratory [28]. Hardware components of the model are: VNLab server, student, teacher and administrator workstations and network infrastructure that enable remote access to the VNLab server. Due to the software components complexity they will be described further in more details.

VNLab is based on Microsoft Virtual Server 2005 R2 [29] virtualization software. At first VNLab is hosted on Microsoft Windows 2003 platform and web server Microsoft IIS 6.0 (Internet Information Server) [30]. Later, at the beginning of summer semester this year, the laboratory has been ported to the new platform with the Microsoft Windows 2008 and IIS7. Its architecture is presented in the Figure 1. Two listed software components create the basis for implementation, administration and utilization of the virtual laboratory. The virtual server 2005 allows creation of virtual machines configurations, as well as its virtual network connections.

The emulated network supports up to 60 virtual machines. This number is sufficient for creation of the virtual network scenarios used for learning networking concepts. Virtual machines emulate hardware and software of physical machines together with the network interface cards (NIC). The maximum number of emulated NICs per virtual machine is four.

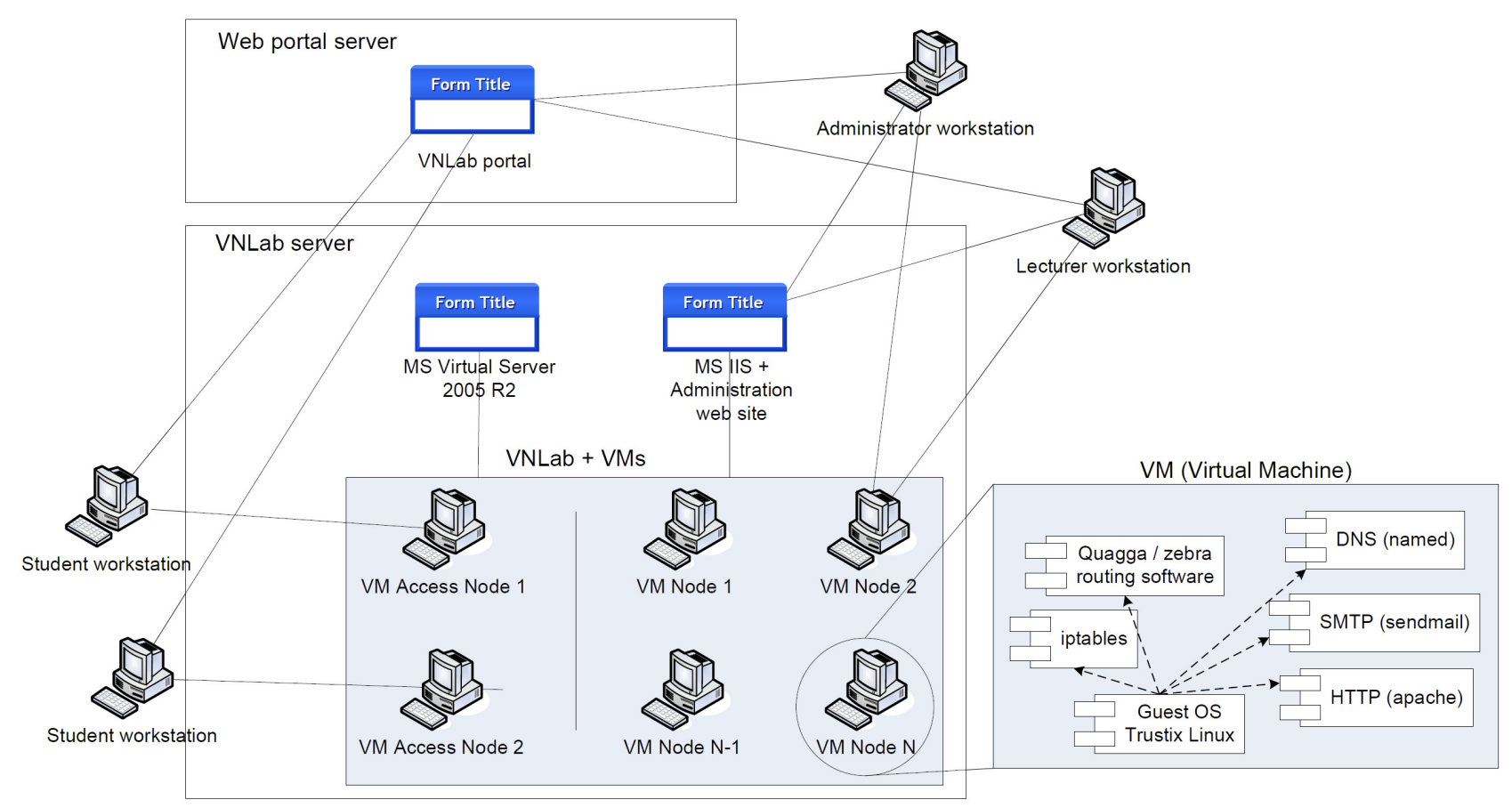

Figure 1. VNLab architecture 
Emulated network has two major types of virtual machines. The first type has a role of computers and routers (nodes), which create the core of the network. Those nodes are preconfigured in order to enable the functional network environment completely similar to the real systems. In the Figure 1 nodes are labeled as VM Node 1, VM Node 2, etc. Emulated nodes are designed to enable creation of virtual network suitable for building the environment for students to learn network concepts and to upgrade their skills with practical work in the real systems. The reality of the emulated network and learning scenarios depends on the configuration of these nodes.

The second type of the nodes (Figure 1) refers to access nodes (VM AccessNode1, VM AccessNode2 etc.). Those nodes have only basic configuration. During the exercise students remotely log on those nodes and configure them in order to make them functional parts of existing network. The configuration depends on the current learning topic. The core of the network must be designed and configured in a way to provide the same functionality for all students during the exercises.

Remote access is provided by Microsoft software Virtual Machine Remote Console (VMRC) client software installed together with Virtual Server 2005 R2. It uses VMRC protocol for remote access to the virtual machines. The configuration of each virtual machine is the same. The Guest OS (operating system) installed on machine is GNU Linux. In the first version of the laboratory virtual machines had Trustix Linux 2.2. In the new, upgraded version of the laboratory, the newer versions of the operating system were installed. There are about 20 virtual machines with CentOS 6.2. operating system, with some experimental Scientific Linux workstations. The new workstations are used together with the old, but still functional machines with Trustix OS. The additional software is defined by multifunctional characteristics of the virtual machines. Depending on the scenario their role is to perform the task of: web, mail, ftp or DNS server, static and/or dynamic routing, firewall or to be a simple workstation.

At the moment, in the VNLab environment exists about 60 virtual machines, but this number is changing constantly. The vital part of the system is its main hardware component - the VNLab server. Further development includes integration of additional OS in the laboratory (e.g. Scientific Linux, Microcore, SMS Linux, NetBSD etc.).

\section{THE LAB EXERCISES IN THE VNLAB}

In order to make VNLab environment usable for Computer Network course and to adjust it to the lectures and curricula of the same course, 12 laboratory exercises are created. Most of exercises consist of three parts: the short introduction, programmed task and problems to solve. The introduction has a brief explanation of the topic to be learned, together with the terms needed for the exercise. The second part, the one with programming task contains step-by-step instruction how to log on the virtual machine and how to configure particular machine. The third part contains problem related to the exercise topic. Students solve this problem by configuring their machines. Only few tasks are not applicable on the virtual machines. Instead, students resolve their tasks on the paper.
Because of the short introductory part which is given at the beginning of the exercise and the concise explanation during the step-by-step configuration instruction, the required starting knowledge for the lab usage is limited only to brief OSI model and TCP/IP protocol basics.

The created exercises are presented in the Table I. There are: the code of the exercise, its name and set of learning topics, terms, concepts and commands covered by the exercises.

TABLE I.

LIST OF VNLAB EXERCISES

\begin{tabular}{|c|c|}
\hline Lab & Lab exercise description \\
\hline \multirow[b]{2}{*}{ VNLab00 } & VNLab environment and LinuxOS \\
\hline & $\begin{array}{l}\text { introductory exercise, VNLab environment, Linux } \\
\text { operating system, Linux file system, network settings, } \\
\text { configuration files, commands: ifconfig }\end{array}$ \\
\hline \multirow[b]{2}{*}{ VNLab01 } & IPv4 addressing and static routing - part 1 \\
\hline & $\begin{array}{l}\text { IPv4 addressing, IP address, netmask, network prefix, } \\
\text { network address, broadcast address, routing introduction, } \\
\text { static routing, commands: route add, route del, route, } \\
\text { netconfig, ping, traceroute. }\end{array}$ \\
\hline \multirow[b]{2}{*}{ VNLab02 } & IPv4 addressing and static routing - part 2 \\
\hline & $\begin{array}{l}\text { IPv4 addressing, IP address, netmask, broadcoast } \\
\text { address, static routing, network design, default route, } \\
\text { commands: route add, route del, route, netconfig, ping, } \\
\text { traceroute. }\end{array}$ \\
\hline \multirow[b]{2}{*}{ VNLab03 } & IPv6 addressing and static routing \\
\hline & $\begin{array}{l}\text { IPv6, IPv6 addresing, global-unicat address, site-local } \\
\text { address, link-local address, IPv6 static routing, 6to } 4 \\
\text { tunneling, ND protocol, commands: route add, route del, } \\
\text { route show, traceroute. }\end{array}$ \\
\hline \multirow[b]{2}{*}{ VNLab04 } & RIP routing protocol \\
\hline & $\begin{array}{l}\text { Dynamic routing, RIP protocol basics, Zebra/quagga } \\
\text { multiprotocol routing software, Cisco IOS-like } \\
\text { environment, router configuration, commands: router } \\
\text { rip, passive interface, network. }\end{array}$ \\
\hline \multirow[b]{2}{*}{ VNLab05 } & OSPF routing protocol - backbone area \\
\hline & $\begin{array}{l}\text { Dynamic routing, OSPF protocol basics, interior } \\
\text { gateway protocols, area 0 (backbone), path costs, } \\
\text { Zebra/quagga multiprotocol routing software, Cisco } \\
\text { IOS-like envirionment, commands: router ospf, passive } \\
\text { interface, network, area,ip ospf cost. }\end{array}$ \\
\hline \multirow[b]{2}{*}{ VNLab06 } & OSPF routing protocol - muliple areas \\
\hline & $\begin{array}{l}\text { Dynamic routing, OSPF protocol basics, interior } \\
\text { gateway protocols, multiple areas, area types, path costs, } \\
\text { Zebra/quagga multiprotocol routing software, Cisco } \\
\text { IOS-like envirionment, commands: router ospf, passive } \\
\text { interface, network, area,ip ospf cost. }\end{array}$ \\
\hline \multirow[b]{2}{*}{ VNLab07 } & BGP routing protocol \\
\hline & $\begin{array}{l}\text { Dynamic routing, BGP protocol basic, exterior gateway } \\
\text { protocols, Zebra/quagga multiprotocol routing software, } \\
\text { Cisco IOS-like environment, autonomus system, } \\
\text { neighbors, commands: router bgp, network, neighbor. }\end{array}$ \\
\hline \multirow[b]{2}{*}{ VNLab08 } & Server software - part 1 \\
\hline & $\begin{array}{l}\text { TCP and UDP protocols and ports, server software, file } \\
\text { letc/services, starting and stopping services, commands: } \\
\text { netstat, telnet; programming simple client and server } \\
\text { software, compiling and installing server software } \\
\text { (source code), rpm packages }\end{array}$ \\
\hline \multirow{2}{*}{ VNLab09 } & Server software - part 2 \\
\hline & $\begin{array}{l}\text { Server software configuration, apache (web server), } \\
\text { proftpd (ftp server), bind (DNS server) }\end{array}$ \\
\hline \multirow[b]{2}{*}{ VNLab10 } & Firewall \\
\hline & $\begin{array}{l}\text { Firewall IPv4 principles, configuration, iptables, packet } \\
\text { filtering, NAT, port forwarding, network and system } \\
\text { security }\end{array}$ \\
\hline \multirow{2}{*}{ VNLab11 } & Firewall IPv6 \\
\hline & $\begin{array}{l}\text { Firewall IPv6 principles, configuration, iptables6, packet } \\
\text { filtering, network and system security }\end{array}$ \\
\hline
\end{tabular}




\section{EXPANSION OF VNLAB USAGE}

The virtual network laboratory is designed for application in the Computer Network course at Information Technology department of Technical Faculty "Mihajlo Pupin" Zrenjanin. Its usability in wider range of network related courses is analyzed by the comparison with recommended curricula's of leading international organization in five areas of computer science [7].

According to the categorization [31] of the leading world computing organizations IEEE (Institute of Electrical and Electronics Engineers), ACM (Association for Computing Machinery) and AIS (Association of Information Systems) in the post 1990 era there are five areas in the field of computer science: Computer Engineering (CE), Computer Science (CS), Information Technology (IT), Information Systems (IS) and Software Engineering (SE). All these categories have integrated course related to computer networks, and the VNLab usability in the recommended curricula was analyzed in the previous research [7].

In the same manner it was started with analyses of possibility of application of VNLab in other course which is not related to networking. The similar methodology of comparison has been used for this analysis.

The starting point of this research in expanding the VNLab usability is to determine the wider areas which can be supported with VNLab. The main criteria for that is VNLab structure and support for variety of programs, as well as the capability of this environment to support the design of completely new lab exercises or adaptation of existing ones for other systems.

The analyses give the overall areas of usability of VNLab in courses related with:

- System security

- Operating systems

- System administration and maintenance

The next phase in the research was to determine computing topics related to the enlisted areas in the recommended curricula's [31]. Five topics are presented in Table II for each area separately.

In table II for each area min and max values are given. The min value represents the minimum emphasis of the topic and the max value represents the greatest emphasis that can typically occur within given curriculum. This report also permits each institution to establish requirements greater than those defined in five curriculum reports [32].

TABLE II

COMPARATIVE WEIGHT OF SECURITY AND ADMINISTRATION TOPICS ACROSS FIVE KINDS OF DEGREE PROGRAMS

\begin{tabular}{|l|c|c|c|c|c|}
\hline Networking and communications & CE & CS & IS & IT & SE \\
\hline $\begin{array}{l}\text { Operating Systems Principles \& } \\
\text { Design }\end{array}$ & $2 / 5$ & $3 / 5$ & $1 / 1$ & $1 / 2$ & $3 / 4$ \\
\hline $\begin{array}{l}\text { Operating Systems Configuration \& } \\
\text { Use }\end{array}$ & $2 / 3$ & $2 / 4$ & $2 / 3$ & $3 / 5$ & $2 / 4$ \\
\hline Security: issues and principles & $2 / 3$ & $1 / 4$ & $2 / 3$ & $1 / 3$ & $1 / 3$ \\
\hline Security: implementation and mgt & $1 / 2$ & $1 / 3$ & $1 / 3$ & $3 / 5$ & $1 / 3$ \\
\hline Systems administration & $1 / 2$ & $1 / 1$ & $1 / 3$ & $3 / 5$ & $1 / 2$ \\
\hline
\end{tabular}

Five extracted areas that correspond to the possible fields of application of VNLab determined in previous chapter are presented in Table II. Description of presented areas is:

Operating Systems Principles \& Design covers principles and design for the system software that manages all hardware resources (including the processor, memory, external storage, and input/output devices).

Operating Systems Configuration \& Use covers skills such as installation, configuration and management of the operating systems on one or more computers.

Security: Issues and Principles deals with access control to computer systems and the information.

Security: Implementation and Management covers organizational activities associated with selection, procurement, implementation, configuration, and management of security processes and technologies for IT infrastructure and applications.

Systems Administration teaches students to manage computing and communications resources, including networks, databases, operating systems, applications, and web delivery. Management issues also include installation, configuration, operation and maintenance [31].

For better illustration, Operating Systems Configuration \& Use have greater importance for Information Technology profile than for computer Science and Software Engineering profiles and even greater importance comparing to Computer Engineering and Information System profiles. The main feature of this topic is that its importance is similar to all areas with only slight variations. The difference is much more recognizable in System administration topic where the greatest importance is given to Informational Technology field comparing to all other fields ( $\min 3$ and $\max 5$ comparing to min 1 and max 1-3).

\section{VNLAB SUPPORT FOR EXPANDED USAGE}

After determination of possible areas of VNLab usage, the next phase in this research was to determine the VNLab capabilities to cover five extracted areas presented in previous section. Again, the VNLab platform and architecture determine this capability. Since the areas of interest are: operating systems, system security and administration, this section discusses current capabilities and possible upgrades of VNLab needed to support these topics. Five extracted areas can be grouped in two main groups considering the similarity of features to be supported in VNLab for these areas. These two groups are System Security and Operating System and System Administration.

\section{A. Application in System Security related courses}

The greatest capability of VNLab is in supporting system security area. The reason for that lies in fact that in standard set of VNLab exercises, already used in Computer Network course (Table I), VNLab has support for teaching firewall system concepts with usage of iptables and netfilter (framework for intercepting and manipulating network packets). Generally, these packages allows stateless packet filtering (IPv4 and IPv6), stateful packet filtering (IPv4 and IPv6) and all kinds of network address and port translation, e.g. NAT/NAPT (IPv4 and IPv6).

With its broad features it can be used for creating variety of learning scenarios different from those used in 
Computer Network courses or the variations of the same scenarios.

The next feature concerning the system security which can be easily implemented in existing VNLab is web security using the SSL (Secure Sockets Layer)/TLS (Transport Layer Security). Since the laboratory is installed with apache server, the lab exercises including the apache web server and configuration of SSL/TLS can be easily designed and implemented in laboratory environment.

The next easily implemented lab exercise is installation and configuration of Secure Shell (SSH) server and client software. Besides the basic installation and configuration, this exercise can be expanded with the example of login authentication, public key and hostbased authentication example. The prototype of this exercise is also implemented in VNLab environment, but it has not been used in the teaching process so far. Besides SSH protocol, the SCP protocol can be presented as well. Also, demonstration of usage and configuration of encryption algorithms such as algorithms: 3DES, Blowfish, AES and ARCFOUR can be supported in this lab exercise.

The last of the related topics in the same security area is demonstration of installation and configuration of proftpd server software with the support for SFTP (Secure file Transfer Protocol) and SCP (Secure Copy).

Further extension of security related learning scenarios can be implemented in the same way as it is in the $V$ Netlab environment [19]. The network traffic tools such as ping, traceroute and nmap can be used for purpose of analysis. The tool snort can be deployed for network intrusion detection as well.

\section{B. Application in Operating System and System Administration related courses}

The complete new area of the VNLab coverage reflects in the domain of operating systems, administration and maintenance. The whole new set of VNLab exercises can be supported in this domain by current configuration and features of VNLab. One of the features related to this field is installation of various client and server software. The installation and configuration of server software is covered with VNLab08 and VNLab09. These lab exercises have already been implemented and tested in laboratory environment. VNLab and its virtual machines with Linux operating system can be used for various tasks.

First, students can install server and client software as rpm or source code packages. After the compilation and installation, student can learn how to start and how to stop services. Next, the initial and basic configuration of installed software can be done by editing its configuration files. In the lab exercises apache web server, proftpd server and sometimes bind dns server have been installed and initially configured so far. This lab exercises can be easily expanded to installation of mail server (sendmail) or some other server as well.

The VNLab and its virtual machines are suitable for studying and practicing operating systems issues like the file system, managing users and user groups and their rights, etc. In its current configuration, the VNLab uses only Linux operating systems (Trustix Linux, CentOS, Scientific Linux, etc), so the presented and supported features are only supported in this environment. Theoreti- cally, its application for studying similar tasks in MS Windows environments is possible, but can be much more demanding because of larger hardware resources required from Microsoft operating system. The second important issue in this case is related to possible difficulties caused with concurrent remote access of about 20 students in the same time to the VNLab server and its impact on network traffic in the case of transferring graphical desktop environments for all 20 virtual machines from VNLab server to student workstations. The same problem may occur in the case of accessing Linux graphical X-windows system, since all presented exercises so far have been designed for work in command line interface. Although the individual access to virtual machines with Microsoft operating system is possible, as well as the performing of administration tasks, it was not tested during the teaching process and for learning in some particular course.

One of the important issues, such as installation of one or multiple operating systems, is also supported in the VNLab environment. For this particular task, students can access virtual machines with empty virtual disks. The operating system installation in the form of iso file can be mounted on virtual CD/DVD drive of virtual machines. In that way students can experience the whole installation process of variety of operating systems.

\section{EXPANDED USABILITY ANALYSES}

After the analyses of VNLab support for other, nonnetwork related courses the set of possible course suitable for integration of VNLab in educational process in different profiles was extracted. In the Computer Engineering curricula there is a subject CE-OPS Operating Systems [33]. In the Information system curricula IS 2010.4 IT Infrastructure as a core course and IT Security and Risk Management as an elective course are suitable for potential VNLab usage [34]. In the Computer Science curricula OS.Operating Systems course with 18 core hours is presented [36]. In Information Technology field following courses are recommended: SA.System Administration and Maintenance (11 core hours), IAS.Information Assurance and Security (23 core hours) and PT. Platform Technologies (14 core hours) [35].

VNLab is applicable in the greatest extent to the subject System Administration and Maintenance (SA) course in Information technology curricula with planned 11 core hours. It consists of four learning units (SA.Operating Systems, SA.Applications, SA.Administrative Activities and SA.Administrative Domains). The aim of this subject is to teach future IT experts those skills and concepts that are essential to the administration of operating systems, networks, software, file systems, file servers, web systems, database systems, and system documentation, policies, and procedures. With the analysis of VNLab structure, architecture and capabilities, this recommended course can be covered in VNLab environment in particular learning topics.

In SA.Operating Systems module these topics are: installation, configuration, maintenance (service packs, patches, etc.) and Server services (print, file, DHCP, DNS, FTP, HTTP, mail, SNMP, telnet). VNLab ability to support this task is presented in previous chapter.

Its core learning outcome covered by VNLab is installation of at least one current operating system. The 
supported advanced learning outcomes are: evaluation of various operating systems and recommendation of a particular operating system to satisfy given needs; modification of the configuration of an operating system; analyzes of pros and cons of installing service packs and updates; recommendation when service packs and operating system updates should be installed; installation of service packs and operating system updates; installation of various server and client services.

In SA.Applications module, the topics covered by VNLab are: installation, configuration, maintenance (service packs, patches, etc.), server services (database, web, network services, etc.) and client services.

Core learning outcomes are: installation of at least one current application; learning how to distinguish between server and client services.

In SA.Administrative Activities module covered topics are: content deployment (file system planning and structure), server administration and management, user and group management, security management and automation management (automatic job scheduling). As it was described in previous chapter, all presented topics are currently applicable for the Linux platform, because this platform is currently used in VNLab environment. Usage of other platforms, such as Microsoft or BSD, is possible but not tested in real teaching process and work with students.

Core supported learning outcomes of this module are: identification of situations in which administrative activities are required and identification of situations which interfere with administrative activities.

Advanced learning outcomes are: identification and explanation of responsibilities associated with server administration and management and explanation of benefits of managing users and groups.

In SA.Administrative Domains topics are: web domain, network domain, OS domain, support domain. First three enlisted domains are easily supported with VNLab.

Core learning outcomes: description of the responsibilities common to the various administrative domains; description of the responsibilities unique to each of the various administrative domains and identification responsibilities in each domain that support activities in other domains.

Advanced learning outcome is justification of how to allocate resources for various administrative domains.

The greatest compatibility of VNLab environment is with the presented course System Administration and Maintenance (SA).

The other subjects such as IAS.Information Assurance and Security can be supported by VNLab, but in this stage of VNLab development, only in small portion. The same is with the PT. Platform Technologies course, where the support is limited to the PT. Operating Systems module. Similar situation is with IS 2010.4 IT Infrastructure course in Information Systems profile, where only one part of the course where the topics concerning operating systems such as file systems and storage, operating system configuration and securing an operating system can be supported by VNLab.

All other courses presented in the beginning of this section are supported with VNLab in a very small quantity.

\section{CONSLUSION AND FURTHER WORK}

VNLab, the virtual network laboratory with remote access, based on virtualization technology, developed at Technical Faculty "Mihajlo Pupin" - Zrenjanin, University of Novi Sad has been tested in the engineering education process and in teaching Computer Network course since 2007. The general usability of VNLab environment is analyzed in teaching network related courses by comparison with recommended undergraduate curricula of international organizations such as IEEE, ACM and IAS [36].

This paper presents extended research in analyses of wider usability of VNLab in other, non-network related courses. Possible courses suitable for incorporation of VNLab and its utilization for lab exercises are courses related to operating systems, system administration and system security.

According to Table II, VNLab structure, its support concerning the needed tools, software packages and technologies (presented in Section VI) and mostly according to the comparison with listed courses from recommended curricula (presented in Section VII), the conclusion is that VNLab is the most applicable for educating Information Technology undergraduate students and particularly in System Administration and Maintenance course. Its application in system security course for the same profile is also possible but in much smaller portion. In order to make VNLab applicable in System security related courses the extended research has to be done. The goal of this future research will be the modeling and implementation of VNLab scenarios for teaching security topics.

At this stage of research, several security related lab exercises have already been implemented in various forms. Firewall system exercises have already been implemented as two exercises within Computer Network courses. These exercises are used and tested in educational process.

Other exercises, such as SSH, STP and SSL/TSL protocol exercises are implemented as prototype exercises and tested only with limited number of students outside of teaching process. The implementation of other technologies and protocols in the VNLab environment is also one direction of further research and development of VNLab (e.g. Site-to-Site IPSec VPN).

\section{REFERENCES}

[1] E. Weingartner, H. vom Lehn, and K. Wehrle, A performance comparison of recent network simulators, Proceedings of the IEEE International Conference on Communications 2009 (ICC2009), Dresden, Germany, 2009.

[2] Comer Douglas E., Internetworking with TCP/IP, Vol. 1: Principles, Protocols, and Architecture, 4th Edition, Prentice Hall Inc, New Jersey, USA, 2000.

[3] A. Wang and W. Jiang, Research of Teaching on Network Course Based on NS-3, First International Workshop on Education Technology and Computer Science (ETCS'09), Vol. 2, pp 629-632, 2009.

[4] S. Papanastasiou, J. Mittag, E. G. Ström, H. Hartenstein, Bridging the Gap Between Physical Layer Emulation and Network Simulation, Proceedings of IEEE Wireless Communications and Networking Conference (WCNC), pp 1-6, April 18-21, Sidney, Australia, 2010

[5] Wainer Gabriel A., Discrete-event Modeling and Simulation - A Practitioner's Approach, Taylor \& Francis Group, Boca Raton, USA, 2009. http://dx.doi.org/10.1201/9781420053371 
[6] Kenneth Hess, Amy Newman, Practical virtualization solutions : virtualization from the trenches, Pearson Education Inc., Prentice Hall, USA, 2010.

[7] D. Dobrilovic, V. Jevtic, Z. Stojanov, B. Odadzic, "Usability of virtual network laboratory in engineering education and computer network course", Proceedings of joined 15th International Conference on Interactive Collaborative Learning and 41st IGIP International Conference on Engineering Pedagogy, September 26 - 28,

[8] Willach, 2012. Kuczborski, A computer network laboratory based on the concept of virtual machines, World Transactions on Engineering and Technology Education 2005, UICEE, Vol.4, No.1, Perth, Australia, 2005

[9] Jeroen van der Ham, Gert Jan Verhoog, Virtual environments for networking experiments "Analytical Network Project" Masters System and Network Administration, University of Amsterdam, Netherlands, http://staff.science.uva.nl/ vdham/research /publica tions/anp-jvdh-gjv.pdf, July 1, 2004.

[10] Kuthonuzo Luruo, Virtual Networking with User-Mode Linux, Linux for you pro, No. 27, March 2005.

[11] Kuthonuzo Luruo, Getting to Virtual Networking Nirvana, Linux for you pro, No. 27, May 2005.

[12] Dobrilović Dalibor, Stojanov Željko, Using virtualization software in operating systems course, Proceedings of the 4th IEEE International Conference on Information Technology: Research and Education - ITRE 2006, pp. 222-226, ISBN: 1-4244-0859-8, Tel Aviv, Israel, 16-19 October 2006, http://dx.doi.org/10.1109/ITRE.2006.3 $\underline{81569}$

[13] Charles Border, The development and deployment of a multi-user, remote access virtualization system for networking, security, and system administration classes, Proceedings of the 38th SIGCSE technical symposium on Computer science education (SIGCSE '07), pp 576-580, ACM New York, NY, USA, 2007.

[14] Xinli Wang, Guy C. Hembroff, Rick Yedica, Using VMware VCenter lab manager in undergraduate education for system administration and network security, Proceeding of ACM conference on Information technology education (SIGITE '10), pp 43-51, Oc-

[15] Kyle E. Stewart, Jeffrey W. Humphries, Todd R. Andel, Developing a virtualization platform for courses in networking, systems administration and cyber security education, Proceedings of the 2009 Spring Simulation Multiconference (SpringSim '09), Society for Computer Simulation International San Diego, CA, USA,

[16] Li $^{2009}$ Yang, Teaching system and network administration using virtual PC, Journal of Computing Sciences in Colleges archive, Vol. 23, Issue 2, Consortium for Computing Sciences in Colleges, USA, December 2007

[17] Bullers, Jr., W. I. , Burd S., Seazzu, A. F., Virtual machines - an idea whose time has returned: application to network, security, and database courses, Proceedings of the 37th SIGCSE technical symposium on Computer science education (SIGSCE'06), pp 102-106,

[18] Menjamin R. Anderson, Amy K. Joines, Thomas E. Daniels, Xen worlds: leveraging virtualization in distance education, Proceedings of Annual Joint Conference Integrating Technology into Computer Science Education (ITiCSE), pp 293-297, Paris, France, July 2009

[19] Sun W., Katta V., Krishna K., Sekar R., V-NetLab: An Approach for Realizing Logically Isolated Networks for Security Experiments, Proceedings of (CSET'08), San Jose, USA, July 28 - August 1,2008 .

[20] Valerie J. H. Powell, Christopher T. Davis, Randall S. Johnson, Peter Y. Wu, John C. Turchek, Ian W. Parker, VLabNet: the integrated design of hands-on learning in information security and networking, Proceedings of the 4th annual conference on Information security curriculum development (InfoSecCD'07), ACM New York, NY,USA, 2007

[21] Valerie J.'H. Powell, John C. Turchek, Peter Y. Wu, Lawrence C. Franzi, Randall S. Johnson, Ian W. Parker, Christopher T. Davis, VLabNet: A Virtual Laboratory Environment for Teaching Networking and Data Communications, Proceedings of ISECON 2007, Vol. 24, pp 1-9, Pittsburgh, USA, 2007.

[22] Muthucumaru Maheswaran, Alexis Malozemoff, Daniel Ng, Sheng Liao, Song Gu, Balasubramaneyam Maniymaran, Julie Raymond McGill, Reehan Shaikh, Yuanyuan Gao, GINI: a userlevel toolkit for creating micro internets for teaching \& learning computer networking, SIGCSE '09 Proceedings of the 40th ACM technical symposium on Computer science education, March 3-7, 2009, Chattanooga, Tennessee, USA, 2009.

[23] B. Kneale, L. Box, A virtual learning environment for real-world networking, Informing Science, pp 71, Pori, Finland, June 2003.

[24] B. Kneale, A. Y. De Horta, I. Box, Velnet: virtual environment for learning networking, Proceedings of the 6th conference on Australian computing education ACE2004, Vol. 30, pp 161 - 168, Dunedin, New Zealand, 2004

[25] Jean-Vincent Loddo, Luca Saiu, Marionnet: a virtual network laboratory and simulation tool, SimulationWorks, March 4, Marseille, France, 2008.

[26] Jiang, X., D. Xu., vBET: a VM-Based Emulation Testbed, Proceedings of the ACM SIGCOMM 2003 Workshops, pp 95104, Karlsruhe, Germany, August, 2003.

[27] Anuzelli G, Dynagen: The Network Configuration Generator for Dynamips, http://dynagen.org, 2007.

[28] Robert Larson, Janique Carbone, Microsoft Virtual Server 2005 R2 Resource Kit Chapter 7: Best Practices for Configuration and Performance, Microsoft Press, Redmond, USA, August, 2007.

[29] Dobrilovic D., Stojanov Z., Odadzic B., Design and Implementation of Online Virtual Network Laboratory, Advancement in Online Education: Exploring the Best Practices, Vol. 1, Chapter 10, Publication date: 2011 4th quarter, 2011

[30] Mitch Tulloch, IIS 6 Administration, McGraw Hill, April, 2003.

[31] Computing Curricula 2005 - The Overview Report, The Joint Task Force for Computing Curricula 2005, A cooperative project of The Association for Computing Machinery (ACM), The Association for Information Systems (AIS), The Computer Society (IEEE-CS), http://www.acm.org/education/education/curric vols/CC2005March06Final.pdf, September 2005.

[32] Barry M. Lunt et al., Information Technology 2008, Curriculum Guidelines for Undergraduate Degree Programs in Information Technology, Association for Computing Machinery (ACM) and IEEE Computer Society, http://www.acm.org//education/curricula/ IT2008\%20Curriculum.pdf, Novembar 2008.

[33] Computer Engineering 2004, Curriculum Guidelines for Undergraduate Degree Programs in Computer Engineering - A Report in the Computing Curricula Series, The Joint Task Force on Computing Curricula, IEEE Computer Society and Association for Computing Machinery, http://www.acm.org/education/education/ curric vols/CE-Final-Report.pdf, December 2004.

[34] Curriculum Guidelines for Undergraduate Degree Programs in Information Systems, Association for Computing Machinery (ACM) and Association for Information Systems (AIS), http://www.acm.org/education/curricula/IS\%202010\%20ACM $\% 2$ 0final.pdf, 2010

\section{AUTHORS}

D. Dobrilovic is with Department of Information Technology University of Novi Sad / Technical Faculty "Mihajlo Pupin", Djure Djakovica bb, 2300 Zrenjanin, Serbia (e-mail: ddobrilo@tfzr.rs)

V. Jevtic is with Department of Information Technology University of Novi Sad / Technical Faculty "Mihajlo Pupin", Djure Djakovica bb, 2300 Zrenjanin, Serbia (e-mail: vesna@tfzr.uns.ac.rs)

B. Odadzic is with Department of Information Technology University of Novi Sad / Technical Faculty "Mihajlo Pupin", Djure Djakovica bb, 2300 Zrenjanin, Serbia (e-mail: borislav.odazic@tfzr.rs)

This work is financially supported by Ministry of Education and Science of the Republic of Serbia under the project number TR32044 "The development of software tools for business process analysis and improvement", 2011-2014. It is an extended and modified version of a paper presented at the International Conference on Interactive Collaborative Learning (ICL2012), held 26 - 28 September 2012, in Villach, Austria. Received 30 November 2012. Published as resubmitted by the authors 24 January 2013. 